PSICOLOGÍA

IBEROAMERICANA
Psicología Iberoamericana ISSN: 1405-0943

revista.psicologia@ibero.mx

Universidad Iberoamericana, Ciudad de México México

\title{
Efectos de la privación de alimento en el desarrollo de la sensibilización locomotora provocada por metanfetamina en ratas
}

\author{
Jiménez, Juan C.; Miranda, Florencio \\ Efectos de la privación de alimento en el desarrollo de la sensibilización locomotora provocada por \\ metanfetamina en ratas \\ Psicología Iberoamericana, vol. 26, núm. 1, 2018 \\ Universidad Iberoamericana, Ciudad de México, México \\ Disponible en: http://www.redalyc.org/articulo.oa?id=133959553010
}




\title{
Efectos de la privación de alimento en el desarrollo de la sensibilización locomotora provocada por metanfetamina en ratas
}

\author{
Effects of food deprivation on the development of \\ methamphetamine-induced locomotor sensitization in rats \\ Juan C. Jiménez \\ Universidad Nacional Autónoma de México , México \\ Florencio Miranda fmirandah@yahoo.com \\ Universidad Nacional Autónoma de México, México
}

Resumen: Actualmente hay pruebas acumuladas que indican que la hipoinsulinemia provocada por un agente diabetogénico reduce las autoadministraciones de anfetamina. Nosotros reportamos previamente que la privación de alimento también reduce la expresión de la sensibilización locomotora inducida por metanfetamina (met). En el presente estudio examinamos los efectos de la privación de alimento en el desarrollo de la sensibilización locomotora provocada por met. Diferentes grupos de ratas fueron tratados con met $(1 \mathrm{mg} / \mathrm{kg})$ o salina durante 5 días después de 24 horas de privación de alimento. En el día de prueba fueron tratados con met o salina. Los resultados mostraron que la administración repetida de met produjo un incremento en la actividad locomotora en las ratas durante la fase de desarrollo de la sensibilización locomotora, independientemente de si estaban o no privadas de alimento. Los resultados del día de prueba mostraron que en ningún grupo se alteró la actividad locomotora, independientemente si las ratas fueron privadas o no durante la fase de desarrollo de la sensibilización locomotora. Estos resultados están en línea con estudios previos que sugieren que la privación de alimento afecta la expresión y no el desarrollo de la sensibilización locomotora inducida por los psicoestimulantes como la cocaína, la anfetamina y la met.

Palabras clave: narrativa, metanfetamina, privación de alimento, sensibilización locomotora.

Abstract: Accumulating evidence suggests that hypoinsulinemia, produced by a diabetogenic agent, reduces amphetamine self-administration. We previously reported that food deprivation also reduces the expression of the methamphetamine (meth)induced locomotor sensitization. In the present study we examine the effects of food deprivation on the development of meth-induced locomotor sensitization. Separate groups of rats were treated with meth $(1 \mathrm{mg} / \mathrm{kg})$ or saline during 5 days after $24 \mathrm{~h}$ of food deprivation. On test day, several groups were treated with meth or saline. The results show that repeated administration of meth led to an increase in locomotor activity in rats on development of locomotor sensitization, regardless of whether they were food deprived or not. The results on the test day show that in any group the locomotor activity was altered, regardless of whether they were food deprived or not during the development of meth-induced locomotor sensitization. These results are in line with those of previous studies that suggest that food deprivation affects the expression, but not the development, of psychostimulant-induced locomotor sensitization such as cocaine, amphetamine and meth.

Keywords: methamphetamine, food deprivation, locomotor sensitization. 


\section{INTRODUCCIÓN}

El abuso de los psicoestimulantes como la cocaína, la anfetamina (anf) y la metanfetamina (met) causan diversos desordenes neuropsiquiátricos que incluyen la adicción a las drogas. La cocaína, la anf y la met son agonistas monoaminérgicos indirectos que tienen afinidad por los transportadores de dopamina ( $\mathrm{da})$, norepinefrina (ne) y serotonina ( 5 -ht $)$ involucrados en la recaptura y almacenamiento vesicular de neurotransmisores (Rothman \& Baumann, 2003). La cocaína es un inhibidor de la recaptura de da, ne y 5-ht (Elliott \& Beveridge, 2005). Por lo tanto, aumenta los niveles sinápticos de esos neurotransmisores. La anf y la met actúan sobre los transportadores de da, ne y 5 -ht en las vesículas sinápticas para promover un aumento en la concentración citoplasmática de estas monoaminas y también invierten la dirección de los transportadores de membrana de las monoaminas, facilitando la salida de los neurotransmisores a la hendidura sináptica (Elliott \& Beveridge, 2005; Kahlig \& Galli, 2003; Rothman \& Baumann, 2003).

El sistema mesolímbico de la da, en particular la proyección del área tegmental ventral (atv) al núcleo accumbens (nAcc), es un importante locus para la producción de las propiedades locomotoras, reforzantes, gratificantes y discriminativas de la cocaína, anf y met (Di Chiara, 1995; Filip \& Cunningham, 2002; Koob, 1992; Pontieri, Tanda, \& Di Chiara, 1995). La administración de anf, met o cocaína aumenta la neurotransmisión de da al interferir la función apropiada del transportador de recaptura de la da (tda) facilitando la señalización daérgica en áreas límbicas (Koob, 1992; Koob \& Bloom, 1988).

Actualmente hay pruebas acumuladas que sugieren que las vías de señalización de la insulina modulan numerosas funciones cerebrales, incluyendo la regulación del tda en los sistemas de la gratificación del cerebro (Daws et al., 2011; Williams et al., 2007). Recientemente, algunos estudios han reportado la capacidad de las vías de señalización de la insulina en el cerebro para regular la función del tda, así como las acciones conductuales de la anf (Daws et al., 2011). De esta forma, algunos estudios han reportado que la hipoinsulinemia atenúa las funciones del tda y, como una consecuencia, reduce también las conductas relacionadas con los psicoestimulantes. Por ejemplo, Galici et al. (2003) reportaron que el agotamiento de la insulina por una inyección de estreptozotocina, un agente diabetogénico, redujo la autoadministración de anf. En un estudio previo de nuestro laboratorio, se reportó que la privación aguda de alimento disminuyó la expresión de la sensibilización locomotora inducida por la administración de met. Al igual que la estreptozotocina, la privación de alimento reduce los niveles sanguíneos de insulina (Carr, 1996) y la actividad del tda (Sevak et al., 2008). En el presente estudio examinamos los efectos de la privación de alimento en el desarrollo de la sensibilización locomotora inducida por met en ratas. El proceso de la sensibilización locomotora producida por psicoestimulantes puede separarse en dos fases diferentes, el desarrollo o inducción y la expresión. En el desarrollo se producen las alteraciones celulares y moleculares que 
inducen la sensibilización locomotora, y en la expresión se producen las consecuencias a largo plazo de los cambios producidos durante el desarrollo de la sensibilización locomotora. Por lo tanto, es importante proveer de información, no sólo de la expresión de la sensibilización locomotora inducida por met, sino también del desarrollo.

\section{MÉTODO}

\section{Animales}

Se utilizaron ratas macho de la cepa Wistar de aproximadamente 120 días de edad y con un peso de 220 a 250 g provenientes del Bioterio General de la Facultad de Estudios Superiores Iztacala-unam. Las ratas se alojaron individualmente en cajas-hogar de acrílico de $30 \times 20 \times 20$ $\mathrm{cm}$, con acceso libre a la comida (Teklad LM485 Rat Diet by Harlan) y bajo un ciclo luz-oscuridad controlado (luz: 6:00am-6:00pm) y a una temperatura ambiente de $21 \pm 1^{\circ} \mathrm{C}$. Los experimentos se llevaron a cabo durante la fase de luz (entre 11:00 am y 13:00 pm). Los procedimientos de cuidado y manejo de animales se realizaron conforme a la Norma Oficial Mexicana (NOM-062-00-1999), titulada Especificaciones técnicas para la producción, cuidado y uso de animales de laboratorio.

\section{Drogas}

La droga utilizada en este estudio fue clorhidrato de metanfetamina (Sigma-Aldrich, St Louis mo, usa). La met se disolvió en solución salina y se inyectó intraperitonealmente (ip) en un volumen de $1 \mathrm{ml} / \mathrm{kg}$.

\section{Aparatos}

La actividad locomotora se midió con un sistema de monitoreo de actividad de campo abierto (Modelo ENV-515: Med Associates, St Albans, vt). Cada caja de plexiglás $(40 \times 40 \times 30 \mathrm{~cm})$ estaba equipada con dos conjuntos de foto receptores de movimiento colocados a $2.5 \mathrm{~cm}$ arriba del piso de la caja de actividad y en paredes opuestas para registrar los movimientos ambulatorios $\mathrm{x}$-y. Se registraron las interrupciones de los fotorreceptores a través de un programa de cómputo para producir la distancia horizontal recorrida (en $\mathrm{cm}$ ), la cual se utilizó como la variable dependiente.

\section{Procedimiento}

El experimento se llevó a cabo en tres fases: habituación a las cajas de actividad motora, desarrollo de la sensibilización motora y día de prueba (ver Figura 1 para la cronología del procedimiento general). En todos los ensayos, las ratas (10 por grupo) se colocaban en la caja de actividad 
durante 10 minutos antes de cada sesión para disminuir la conducta exploratoria. Una vez transcurridos los 10 minutos, las ratas fueron regresadas a sus cajas-hogar para la administración de los compuestos dependiendo del grupo al que pertenecían. En el día 2, las ratas fueron introducidas en las cajas de actividad durante 60 minutos (sesión de habituación). En el día 1, las ratas recibieron una inyección ip de $1 \mathrm{ml} /$ $\mathrm{kg}$ de salina, después fueron introducidas en las cajas de actividad motora durante 60 minutos para obtener el registro de la actividad motora (línea base). En los días 1, 3, 5, 7 y 9 las ratas de los grupos M24M y M0M fueron tratados con met $(1 \mathrm{mg} / \mathrm{kg})$ durante el desarrollo de la sensibilización locomotora y la sesión de prueba. Las ratas del grupo M24M se privaron de alimento 24 horas antes de los días 1, 3, 5, 7 y 9. Los grupos S0M, S0S y S24S fueron tratados con salina durante la fase de desarrollo de la sensibilización locomotora y la sesión de prueba, excepto el grupo SOM que fue tratado con met $(1 \mathrm{mg} / \mathrm{kg})$ en la sesión de prueba. Las ratas del grupo S24S se privaron de alimento 24 horas antes de los días 1, 3, 5, 7 y 9. Para todos los grupos, la actividad conductual se registró durante 60 minutos (ver Tabla 1 para diseño experimental).

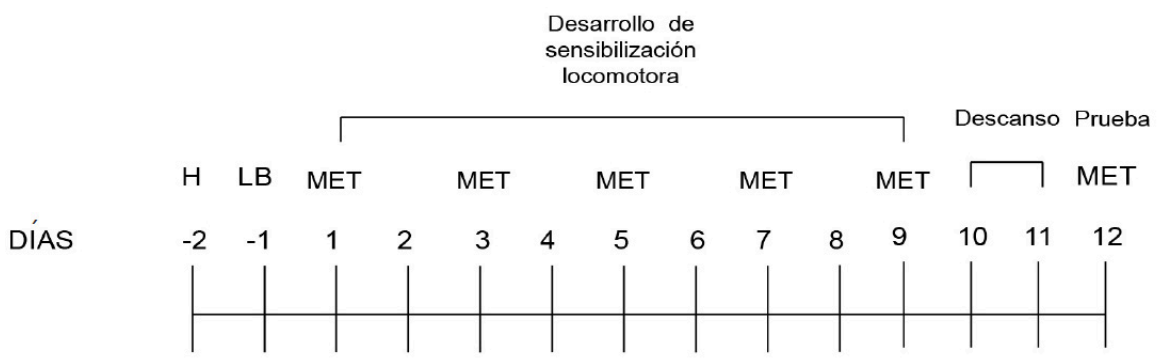

Figura 1

Desarrollo de sensibilización locomotora

Esquema que ilustra la cronología del procedimiento de desarrollo y prueba de la sensibilización locomotora inducida por met, la cual se administró en los días 1, 3, 5, 7 y 9. Algunas ratas se privaron de alimento 24 horas antes de los días 1, 3, 5, 7 y 9 (ver Tabla 1 y la sección de procedimiento para mayor detalle).

\section{Análisis de datos}

Los resultados de la variable investigada (distancia recorrida en $\mathrm{cm}$ ) fueron expresados como la media \pm el error estándar de la media (eem). Los datos obtenidos durante el desarrollo de la sensibilización motora fueron analizados con un anova factorial de medidas repetidas con Días como el factor de medidas repetidas y Grupos como el factor entre sujetos. Los datos obtenidos en la línea base y en la prueba de la sensibilización locomotora inducida por met se analizaron con un anova de una vía. Cuando éstos fueron significativos, se llevó a cabo un análisis de comparaciones posteriores con la prueba de Tukey. En todas las pruebas, el nivel de rechazo del error tipo I fue de .05. 
Tabla 1

Diseño experimental

\begin{tabular}{c|c|c}
\hline Grupos & $\begin{array}{c}\text { Desarrollo } \\
\text { Sensibilidad y privación }\end{array}$ & Prueba \\
\hline M24M & MET (24h) & MET \\
\hline MOM & MET (Oh) & MET \\
\hline SOM & Salina (Oh) & MET \\
\hline SOS & Salina (Oh) & Salina \\
\hline S24S & Salina (24h) & Salina \\
\hline
\end{tabular}

Nota: Metanfetamina $(1.0 \mathrm{mg} / \mathrm{kg})$

Tabla 1

Diseño experimental

\section{RESULTADOS}

Los datos obtenidos durante la línea base de la actividad locomotora fueron similares en todos los grupos $[\mathrm{F}(4,45)=.896, \mathrm{p}=.474]$. La administración repetida de met produjo el desarrollo de sensibilización en la actividad locomotora en los grupos M0M y M24M (Figura 2), mientras que la administración repetida de salina no alteró la actividad motora en los grupos S0M, S0S y S24M. Un anova factorial de medidas repetidas indicó diferencias significativas en los factores Grupos $[\mathrm{F}(4,45)$ $=23.499, \mathrm{p}=.001]$, Días $[\mathrm{F}(1,45)=16.541, \mathrm{p}=.001]$ y de la interacción Grupos x Días $[\mathrm{F}(4,45)=8.609, \mathrm{p}=.001]$. La prueba de Tukey reveló que los grupos M0M y M24M tuvieron diferencias significativas entre los días 1 y 9. Los resultados de la prueba de sensibilización locomotora del día 12 se muestran en la Figura 3. Los resultados mostraron que la administración de met produjo un incremento en la actividad locomotora en los grupos S0M, M0M y M24M [F(4,45) = 40.582, p .0001]. La prueba de Tukey reveló que los grupos M0M y M24M fueron diferentes de los otros grupos. Sin embargo, no se encontraron diferencias significativas entre estos grupos. 


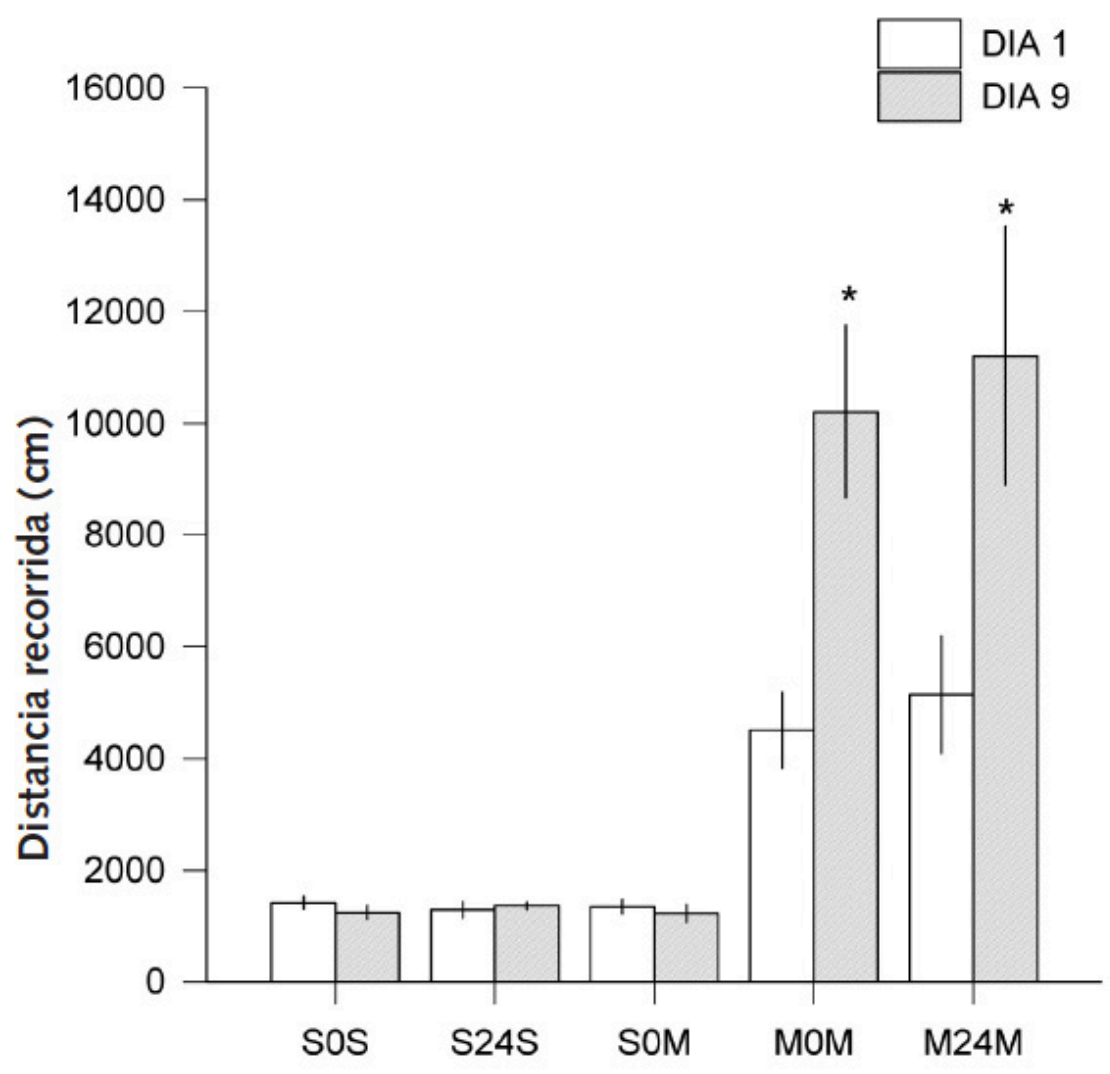

Figura 2

Figura 2

Figura 2 se muestran los resultados de los días 1 y 9 del desarrollo de la sensibilización locomotora inducida por met $(1 \mathrm{mg} / \mathrm{kg})$. La met o salina se administraron a las ratas durante 5 días (1, 3, 5, 7 y 9). Las barras representan la media \pm eem de 10 ratas y los asteriscos indican diferencias significativas con el día 1 respecto a su propio grupo, reveladas por un anova factorial de medidas repetidas seguido por la prueba de comparaciones posteriores Tukey $(\mathrm{p}<0.05)$. 


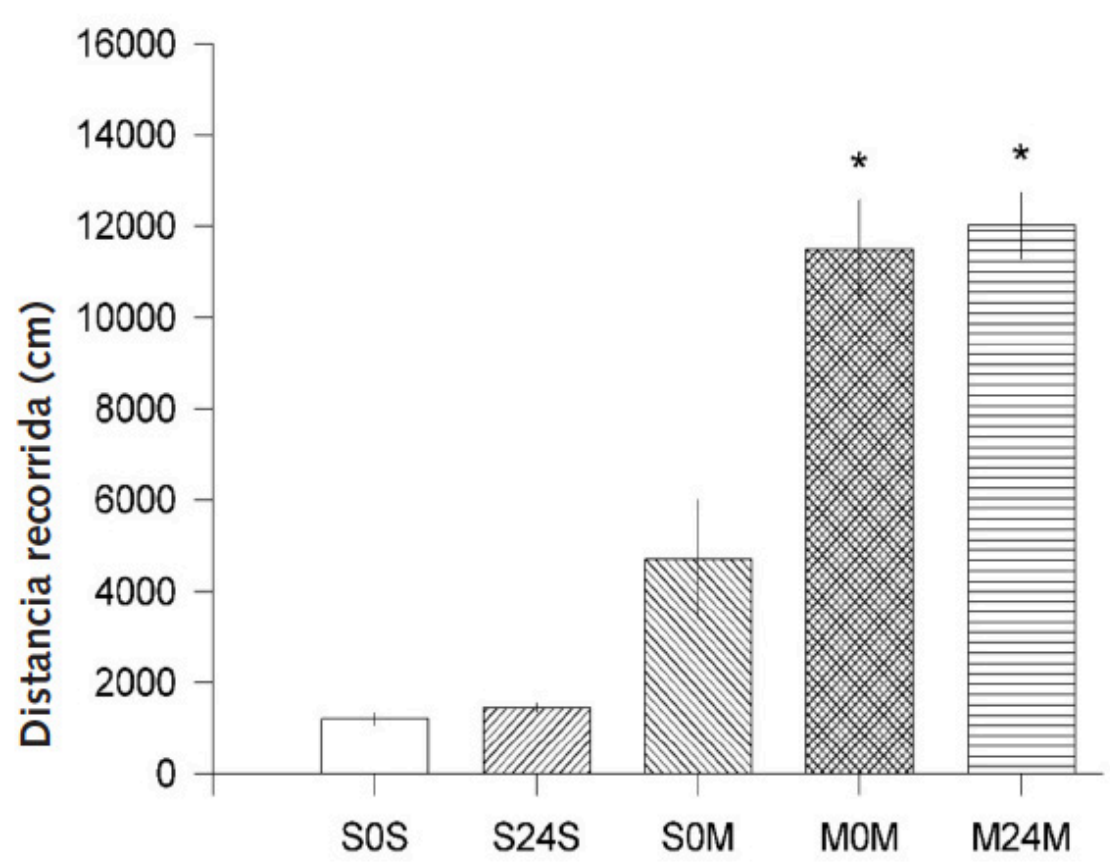

Figura 3

Figura 3

La Figura 3 prueba del desarrollo de la sensibilización locomotora inducida por met en el día 12 . A las ratas se les inyectó $1 \mathrm{mg} / \mathrm{kg}$. Las barras representan la media \pm eem de 10 ratas y los asteriscos indican diferencias significativas con los grupos S0S, S24S y S0M reveladas por un anova de una vía seguido por la prueba de comparaciones posteriores Tukey $(\mathrm{p}<.05)$.

\section{DISCUSIÓN}

El propósito de la presente investigación fue examinar los efectos de la privación de alimento en el desarrollo de la sensibilización locomotora inducida por met en ratas. Observamos que la administración repetida aumentó la actividad locomotora y que la privación de alimento no afectó el desarrollo de la sensibilización locomotora.

Los resultados conductuales producidos por la administración de met son consistentes con estudios previos que demuestran que las ratas que son inyectadas durante varios días con $1 \mathrm{mg} / \mathrm{kg}$ exhiben una actividad locomotora mayor que las ratas tratadas con salina (Hall, Stanis, Avila, \& Gulley, 2008; Kufahl, Nemirovsky, Watterson, Zautra, \& Olive, 2013; Wearne et al., 2015; Zhao et al., 2014). En el presente estudio, también se observó que la privación de alimento no afectó el desarrollo de la sensibilización locomotora inducida por met. La privación de alimento fue programada para algunos grupos (S24S y M24M). Estos resultados son consistentes con estudios previos que reportaron que la restricción 
de alimento (por ejemplo, $10 \mathrm{~g} /$ día) no afecta la locomoción inducida por anf (Sevak et al., 2008) e, incluso, otros han reportado que la restricción de alimento aumenta la actividad locomotora inducida por anf (Stuber, Evans, Higgins, Pu, \& Figlewicz, 2002) y las propiedades reforzantes de la cocaína (Macenski \& Meisch, 1999). En contraste a los resultados del presente estudio y a los resultados mencionados antes, algunos estudios han reportado que la restricción de alimento juega un papel importante en los efectos conductuales de las drogas de abuso. Por ejemplo, se ha reportado que la restricción de alimento redujo el condicionamiento de preferencia de lugar inducido por anf (Stuber et al., 2002). Nosotros hemos reportado que la privación de alimento redujo la expresión de la sensibilización locomotora provocada por met. La razón de esta discrepancia puede estar relacionada con dos fuentes. En la primera, los procedimientos que se emplean en la restricción de alimento son diferentes a los empleados en la privación de alimento. En la restricción el consumo diario se limita a un porcentaje del alimento que consumen los animales en condiciones ad libitum. Esta condición de restricción comienza al menos una semana antes del inicio de las sesiones experimentales o de prueba (Sevak et al., 2008). En el caso de la privación de alimento, los animales se mantienen por varios días con acceso a la comida ad libitum y se privan por cierto periodo de tiempo, por ejemplo, 24 horas, antes de las pruebas bioquímicas o comportamentales (Patterson et al., 1998). Esto podría sugerir que los procedimientos de privación de alimento reducen la expresión de la sensibilización locomotora provocada por psicoestimulantes y la restricción de alimento o no afectan, o incrementan la sensibilización locomotora.

En la segunda, podría involucrar a componentes corriente abajo de las vías de señalización de la insulina cerebral. Por ejemplo, se ha demostrado que la proteína quinasa Akt, componente central de las vías de señalización de la insulina (Hanada, Feng, \& Hemmings, 2004), regula varias funciones cerebrales incluyendo el crecimiento y la supervivencia celular y participa en la regulación de la señalización de la da. Algunos han reportado que la inhibición de la quinasa Akt reduce dramáticamente la depuración de la da y la expresión del tda en la superficie celular (Beaulieu, Gainetdinov, \& Caron, 2009; Speed et al., 2010). Adicionalmente, se ha reportado que la quinasa Akt es necesaria para la expresión, pero no para el desarrollo, de la sensibilización locomotora provocada por psicoestimulantes como la cocaína (Izzo, Martin-Fardon, Koob, Weiss, \& Sanna, 2002). Lo anterior podría explicar porque en el presente estudio la privación de alimento, que disminuye los niveles de insulina, no afectó el desarrollo de la sensibilización locomotora inducida por met.

El mecanismo subyacente en los efectos de la privación de alimento sobre la sensibilización locomotora podría involucrar la participación de las vías de señalización de la insulina en los efectos conductuales de los psicoestimulantes. Algunas líneas de evidencia apoyan esta sugerencia. En primera, las drogas psicoestimulantes tipo anf son agonistas DAérgicos indirectos que tienen afinidad por el tda (Rothman \& Baumann, 2003) e inhiben competitivamente la recaptura de da aumentando la 
concentración extracelular (Khoshbouei, Wang, Lechleiter, Javitch, \& Galli, 2003). En la segunda línea de evidencia, las drogas tipo anf aumentan la liberación de da agotando las vesículas sinápticas de da y promoviendo su liberación invirtiendo la función del tda (Fleckenstein, Volz, Riddle, Gibb, \& Hanson, 2007; Sulzer, 2011). Además, disminuyen la expresión del tda en la superficie celular (Fleckenstein, Metzger, Wilkins, Gibb, \& Hanson, 1997; Kahlig et al., 2006; Saunders et al., 2000). En la tercera línea de evidencia, se ha reportado que las vías de la señalización de la insulina modulan el tráfico y actividad del tda (Daws et al., 2011). La insulina cruza la barrera hematoencefálica y actúa sobre receptores de insulina en áreas límbicas del cerebro como el estriado ventral (Schulingkamp, Pagano, Hung, \& Raffa, 2000), una región del cerebro que expresa el tda (Figlewicz, Evans, Murphy, Hoen, \& Baskin, 2003). Algunos estudios han reportado que el agotamiento de la insulina por la administración de estreptozotocina, una toxina que destruye las células beta del páncreas (Dulin \& Soret, 1977), reduce la actividad del tda (Owens et al., 2005; Sevak et al., 2007), la expresión del tda en la superficie celular (Williams et al., 2007) y la autoadministración de anf (Galici et al., 2003). Al igual que el agotamiento de insulina por la estreptozotocina, la restricción y privación de alimento también reducen los niveles sanguíneos de insulina (Carr, 1996) y la actividad de tda (Patterson et al., 1998; Sevak et al., 2008; Zhen, Reith, \& Carr, 2006). En la cuarta línea de evidencia, la inhibición de PI3K (Izzo et al., 2002) o de Akt, proteínas cinasas corriente abajo de la activación de los receptores de insulina, produce una reducción en la capacidad de la insulina para modular la redistribución del tda en la superficie celular provocada por la anf (Garcia et al., 2005). En línea con lo anterior, se ha reportado que se requiere la integridad de PI3K en la expresión, pero no en el desarrollo, de la sensibilización locomotora provocada por la cocaína (Izzo et al., 2002). Como mencionamos arriba, la sensibilización locomotora refleja neuroadaptaciones que contribuyen a la adicción a las drogas y modelan algunos aspectos de las conductas adictivas como el deseo por la droga (Robinson \& Berridge, 1993). Durante el desarrollo de la sensibilización locomotora ocurren las alteraciones celulares y moleculares que inducen los cambios locomotores y durante la expresión se manifiestan los cambios a largo plazo de esas alteraciones. Al parecer, así lo indican los datos experimentales, la alteración de las vías de la insulina, ya sea por la administración de agentes farmacológicos o por la privación de alimento, sólo alteran la expresión, pero no el desarrollo, de la sensibilización locomotora provocada por la administración de psicoestimulantes. Aunque no está clara aún la causa de estos efectos diferenciales, Izzo et al. (2002) sugieren que la inhibición de PI3K, y como consecuencia de Akt, proteína inmediatamente corriente abajo de PI3K, podría interferir con la expresión de la sensibilización locomotora por alterar la asociación contextual de los estímulos presentes en el desarrollo de la sensibilización locomotora y los presentes durante la prueba de expresión de la sensibilización locomotora. Sin embargo, aún se necesitan pruebas adicionales que fortalezcan esta sugerencia. 
Los resultados de este experimento, junto con los de otros, son un paso importante en el entendimiento de las interacciones entre la insulina, el sistema mesolímbico de la da y los efectos conductuales de los psicoestimulantes y demuestran que la privación de alimento afecta la expresión, pero no el desarrollo de la sensibilización locomotora, lo que podría sugerir que un tratamiento de la adicción a los psicoestimulantes basado en la manipulación del acceso a alimento, sólo sería efectivo una vez que se desarrolló la adicción.

Los próximos estudios sobre esta área tendrán que examinar a detalle el o los mecanismos por los cuales las vías de señalización de la insulina disminuyen los efectos conductuales de los psicoestimulantes. Además, también se deberá comenzar a estudiar la relación entre diabetes, obesidad y adicción a las drogas de abuso.

\section{Agradecimientos}

Esta investigación fue financiada por PAPIIT-UNAM (México) IN307414. Los autores declaran no tener conflictos de interés.

\section{REFERENCIAS}

Beaulieu, J. M., Gainetdinov, R. R., \& Caron, M. G. (2009). Akt/GSK3 signaling in the action of psychotropic drugs. Annual Review of Pharmacology and Toxicology, 49, 327-347. https://doi.org/10.1146/ann urev.pharmtox.011008.145634

Carr, K. D. (1996). Feeding, drug abuse, and the sensitization of reward by metabolic need. Neurochemical Research, 21(11), 1455-1467.

Daws, L. C., Avison, M. J., Robertson, S. D., Niswender, K. D., Galli, A., \& Saunders, C. (2011). Insulin signaling and addiction. Neuropharmacology, 61(7), 1123-1128. https://doi.org/10.1016/j.neuropharm.2011.02.028

Di Chiara, G. (1995). The role of dopamine in drug abuse viewed from the perspective of its role in motivation. Drug \& Alcohol Dependence, 39(2), 95-137. https://doi.org/10.1016/0376-8716(95)01118-I

Dulin, W. E., \& Soret, M. G. (1977). Chemically and hormonally induced diabetes. En B. W. Volk \& K. F. Wellman (Eds.), The diabetic pancreas (pp. 425-465). Boston, MA, US: Springer.

Elliott, J. M., \& Beveridge, T. J. (2005). Psychostimulants and monoamine transporters: Upsetting the balance. Current Opinion in Pharmacology, 5(1), 94-100. https://doi.org/10.1016/j.coph.2004.09.005

Figlewicz, D. P., Evans, S. B., Murphy, J., Hoen, M., \& Baskin, D. G. (2003). Expression of receptors for insulin and leptin in the ventral tegmental area/substantia nigra (VTA/SN) of the rat. Brain Research, 964(1), 107-115. https://doi.org/10.1016/S0006-8993(02)04087-8

Filip, M., \& Cunningham, K. A. (2002). Serotonin 5-HT2C receptors in nucleus accumbens regulate expression of the hyperlocomotive and discriminative stimulus effects of cocaine. Pharmacology Biochemistry and Behavior, 71(4), 745-756. https://doi.org/10.1016/S0091-3057(01)007 41-9 
Fleckenstein, A. E., Metzger, R. R., Wilkins, D. G., Gibb, J. W., \& Hanson, G. R. (1997). Rapid and reversible effects of methamphetamine on dopamine transporters. Journal of Pharmacology and Experimental Therapeutics, 282(2), 834-838.

Fleckenstein, A. E., Volz, T. J., Riddle, E. L., Gibb, J. W., \& Hanson, G. R. (2007). New insights into the mechanism of action of amphetamines. Annual Review of Pharmacology and Toxicology, 47, 681-698. https://doi .org/10.1146/annurev.pharmtox.47.120505.105140

Galici, R., Galli, A., Jones, D. J., Sanchez, T. A., Saunders, C., Frazer, A., Gould, G., Lin, R., \& France, C. P. (2003). Selective decreases in amphetamine self-administration and regulation of dopamine transporter function in diabetic rats. Neuroendocrinology, 77(2), 132-140. https://doi.org/10.11 59/000068650

Garcia, B. G., Wei, Y., Moron, J. A., Lin, R. Z., Javitch, J. A., \& Galli, A. (2005). Akt is essential for insulin modulation of amphetamine-induced human dopamine transporter cell-surface redistribution. Molecular Pharmacology, 68(1), 102-109. https://doi.org/10.1124/mol.104.00909 2

Hall, D. A., Stanis, J. J., Avila, H. M., \& Gulley, J. M. (2008). A comparison of amphetamine and methamphetamine-induced locomotor activity in rats: Evidence for qualitative differences in behavior. Psychopharmacology, 195(4), 469-478. https://doi.org/10.1007/s00213-007-0923-8

Hanada, M., Feng, J., \& Hemmings, B. A. (2004). Structure, regulation and function of $\mathrm{PKB} / \mathrm{AKT}$ - a major therapeutic target. Biochimica et Biophysica Acta (BBA) - Proteins and Proteomics, 1697(1-2), 3-16. https:/ /doi.org/10.1016/j.bbapap.2003.11.009

Izzo, E., Martin-Fardon, R., Koob, G. F., Weiss, F., \& Sanna, P. P. (2002). Neural plasticity and addiction: PI3-kinase and cocaine behavioral sensitization. Nature Neuroscience, 5(12), 1263-1264. https://doi.org/10.1038/nn977

Kahlig, K. M., \& Galli, A. (2003). Regulation of dopamine transporter function and plasma membrane expression by dopamine, amphetamine, and cocaine. European Journal of Pharmacology, 479(1-3), 153-158. https ://doi.org/10.1016/j.ejphar.2003.08.065

Kahlig, K. M., Lute, B. J., Wei, Y., Loland, C. J., Gether, U., Javitch, J. A., \& Galli, A. (2006). Regulation of dopamine transporter trafficking by intracellular amphetamine. Molecular Pharmacology, 70(2), 542-548. https://doi.org/ 10.1124/mol.106.023952

Khoshbouei, H., Wang, H., Lechleiter, J. D., Javitch, J. A., \& Galli, A. (2003). Amphetamine-induced dopamine efflux. A voltage-sensitive and intracellular $\mathrm{Na}$-dependent mechanism. Journal of Biological Chemistry, 278(14), 12070-12077. https://doi.org/10.1074/jbc.M212815200

Koob, G. F. (1992). Drugs of abuse: Anatomy, pharmacology and function of reward pathways. Trends in Pharmacological Sciences, 13(5),177-184. htt ps://doi.org/10.1016/0165-6147(92)90060-J

Koob, G. F., \& Bloom, F. E. (1988). Cellular and molecular mechanisms of drug dependence. Science, 242(4879), 715-723. https://doi.org/10.1126/scien ce. 2903550

Kufahl, P. R., Nemirovsky, N. E., Watterson, L. R., Zautra, N., \& Olive, M. F. (2013). Positive or negative allosteric modulation of metabotropic glutamate receptor 5 (mGluR5) does not alter expression of behavioral 
sensitization to methamphetamine. F1000Research, 2(84). https://doi.or g/10.12688/f1000research.2-84.v1

Macenski, M., \& Meisch, R. A. (1999). Cocaine self-administration under conditions of restricted and unrestricted food access. Experimental and Clinical Psychopharmacology, 7(4), 324-337. http://dx.doi.org/10.1037/ 1064-1297.7.4.324

Owens, W. A., Sevak, R. J., Galici, R., Chang, X., Javors, M. A., Galli, A., France, C. P., \& Daws, L. C. (2005). Deficits in dopamine clearance and locomotion in hypoinsulinemic rats unmask novel modulation of dopamine transporters by amphetamine. Journal of Neurochemistry, 94(5), 1402-1410. https://doi.org/10.1111/j.1471-4159.2005.03289.x

Patterson, T. A., Brot, M. D., Zavosh, A., Schenk, J. O., Szot, P., \& Figlewicz, D. P. (1998). Food deprivation decreases mRNA and activity of the rat dopamine transporter. Neuroendocrinology, 68(1), 11-20. https://doi.org $/ 10.1159 / 000054345$

Pontieri, F. E., Tanda, G., \& Di Chiara, G. (1995). Intravenous cocaine, morphine, and amphetamine preferentially increase extracellular dopamine in the" shell" as compared with the "core" of the rat nucleus accumbens. Proceedings of the National Academy of Sciences, 92(26),12304-12308. https://doi.org/10.1073/pnas.92.26.12304

Robinson, T. E., \& Berridge, K. C. (1993). The neural basis of drug craving: An incentive-sensitization theory of addiction. Brain Research Reviews, 18(3), 247-291. https://doi.org/10.1016/0165-0173(93)90013-P

Rothman, R. B., \& Baumann, M. H. (2003). Monoamine transporters and psychostimulant drugs. European Journal of Pharmacology, 479(1-3), 23-40. https://doi.org/10.1016/j.ejphar.2003.08.054

Saunders, C., Ferrer, J. V., Shi, L., Chen, J., Merrill, G., Lamb, M. E., Leeb-Lundberg, L. M., Carvelli, L., Javitch, J. A., \& Galli, A. (2000). Amphetamine-induced loss of human dopamine transporter activity: An internalization-dependent and cocaine-sensitive mechanism. Proceedings of the National Academy of Sciences, 97(12), 6850-6855. https://doi.org/ $10.1073 /$ pnas. 110035297

Schulingkamp, R. J., Pagano, T. C., Hung, D., \& Raffa, R. B. (2000). Insulin receptors and insulin action in the brain: Review and clinical implications. Neuroscience \& Biobehavioral Reviews, 24(8), 855-872. https://doi.org/1 0.1016/S0149-7634(00)00040-3

Sevak, R. J., Koek, W., Owens, W. A., Galli, A., Daws, L. C., \& France, C. P. (2008). Feeding conditions differentially affect the neurochemical and behavioral effects of dopaminergic drugs in male rats. European Journal of Pharmacology, 592(1-3), 109-115. https://doi.org/10.1016/j.ejphar.20 08.07.002

Sevak, R. J., Owens, W. A., Koek, W., Galli, A., Daws, L. C., \& France, C. P. (2007). Evidence for D2 receptor mediation of amphetamine\# induced normalization of locomotion and dopamine transporter function in hypoinsulinemic rats. Journal of Neurochemistry, 101(1), 151-159. htt ps://doi.org/10.1111/j.1471-4159.2006.04358.x

Speed, N. K., Matthies, H. J., Kennedy, J. P., Vaughan, R. A., Javitch, J. A., Russo, S. J., Lindsley, C. W., Niswender, K., \& Galli, A. (2010). Aktdependent and isoform-specific regulation of dopamine transporter cell 
surface expression. ACS Chemical Neuroscience, 1(7), 476-481. https://d oi.org/10.1021/cn100031t

Stuber, G. D., Evans, S. B., Higgins, M. S., Pu, Y., \& Figlewicz, D. P. (2002). Food restriction modulates amphetamine\#conditioned place preference and nucleus accumbens dopamine release in the rat. Synapse, 46(2), 83-90. https://doi.org/10.1002/syn.10120

Sulzer, D. (2011). How addictive drugs disrupt presynaptic dopamine neurotransmission. Neuron, 69(4), 628-649. https://doi.org/10.1016/j.n euron.2011.02.010

Wearne, T. A., Mirzaei, M., Franklin, J. L., Goodchild, A. K., Haynes, P. A., \& Cornish, J. L. (2015). Methamphetamine-induced sensitization is associated with alterations to the proteome of the prefrontal cortex: Implications for the maintenance of psychotic disorders. Journal of Proteome Research, 14(1), 397-410. https://doi.org/10.1021/pr500719f

Williams, J. M., Owens, W. A., Turner, G. H., Saunders, C., Dipace, C., Blakely, R. D., France, C. P., Gore, J. C., Daws, L. C., Avison, M. J., \& Galli, A. (2007). Hypoinsulinemia regulates amphetamine-induced reverse transport of dopamine. PLoS Biology, 5(10), e274. https://doi.or g/10.1371/journal.pbio.0050274

Zhao, Z., Kim, Y. W., Yang, Y., Zhang, J., Jung, J. Y., Chang, S., Cho, I., Zhou, F., Zhao, J., Lee, B., Yang, C., Kim, S., \& Zhao, R. (2014). Glycyrrhizae radix methanol extract attenuates methamphetamineinduced locomotor sensitization and conditioned place preference. Evidence-Based Complementary and Alternative Medicine, 1-7. http://dx. doi.org/10.1155/2014/152063

Zhen, J., Reith, M. E., \& Carr, K. D. (2006). Chronic food restriction and dopamine transporter function in rat striatum. Brain Research, 1082(1), 98-101. https://doi.org/10.1016/j.brainres.2006.01.094 